九州大学学術情報リポジトリ

Kyushu University Institutional Repository

\title{
Stochastic analysis and the KdV equation
}

Taniguchi, Setsuo

Faculty of Mathematics, Kyushu University

http://hdl. handle. net/2324/3397

出版情報: MHF Preprint Series. 2006-30，2006-11-17. 九州大学大学院数理学研究院 バージョン：

権利関係 : 


\section{MHF Preprint Series}

Kyushu University

21st Century COE Program

Development of Dynamic Mathematics with

High Functionality

\section{Stochastic analysis and the $\mathrm{KdV}$ equation}

S. Taniguchi

MHF 2006-30

( Received November 17, 2006 )

Faculty of Mathematics

Kyushu University

Fukuoka, JAPAN 


\title{
Stochastic analysis and the KdV equation
}

\author{
Setsuo Taniguchi \\ Dedicated to Professor Yoichiro Takahashi on the occasion of his 60th birthday

\begin{abstract}
N. Ikeda and the author established a mapping from a class of Gaussian measures parameterized by linear combinations of Dirac measures on $\mathbb{R}$ to that of reflectionless potentials. The bijectivity of the mapping was shown by the author, and was extended to a class of more general Gaussian measures.

In this paper, a brief review on the bijection and its application to the $\mathrm{KdV}$ equation is given first. Next another application to the stochastic KdV equation is discussed. Finally presented is an alternative approach to the bijection via the linear filtering theory.
\end{abstract}

\section{Introduction}

Applications of stochastic analysis to the theory of partial differential equations (PDE in short) have their source in stochastic representations of solutions; let $\mathcal{L}^{V}$ be a second order differential operator on $\mathbb{R}^{n}$ of the form

$$
\mathcal{L}^{V}=\frac{1}{2} \sum_{i, j=1}^{n} a^{i j} \frac{\partial^{2}}{\partial x_{i} \partial x_{j}}+\sum_{i=1}^{n} b^{i} \frac{\partial}{\partial x_{i}}+V
$$

where $a^{i j}, b^{i}, 1 \leq i, j \leq n$, and $V$ are appropriately smooth functions from $\mathbb{R}^{n}$ to $\mathbb{R}$. The solution $u=u(x, t)$ of the Cauchy problem of the PDE

is represented as

$$
\frac{\partial u}{\partial t}=\mathcal{L}^{V} u, \quad u(\cdot, 0)=f
$$

$$
u(x, t)=\mathbb{E}\left[f(X(t, x)) \exp \left(\int_{0}^{t} V(X(s, x)) d s\right)\right]
$$

where $\{X(t, x)\}_{t \geq 0}$ is the diffusion process stating from $x$ at time 0 , which is generated by $\mathcal{L}^{0}$, and $\mathbb{E}$ stands for the expectation with respect to the underling probability measure. This kind of expression goes back to the studies in 1940's made

2000 Mathematics Subject Classification. Primary 60H30; 60B10; Secondary 34L25.

Key words and phrases. Wiener integral, Reflectionless potential, KdV equation, Linear filtering theory.

Research supported in part by Grant-in-Aid for Scientific Research (A) 14204010. 
by K. Itô $[\mathbf{I t}]$, R. Cameron-W. Martin $[\mathbf{C M}]$, and M. Kac $[\mathbf{K a}]$. In this paper, we shall first give a review on such expressions for classical and generalized reflectionless potentials, and $n$-soliton solutions of the Korteweg-de Vries (KdV in short) equation

$$
\frac{\partial v}{\partial t}=\frac{3}{2} v \frac{\partial v}{\partial x}+\frac{1}{4} \frac{\partial^{3} v}{\partial x^{3}} .
$$

Secondly, we shall apply our probabilistic expression to the stochastic KdV equation observed in $[\mathbf{W}]$. Finally we shall give an alternative proof of the probabilistic expressions of reflectionless potentials by using the filtering theory.

The author is grateful to Professors Gui-Qiang Chen, Elton Hsu, and Mark Pinsky for the stimulating conference at the Northwestern University in June, 2005, and their hospitality.

\section{Reflectionless potentials}

Let

$$
\mathcal{S}=\left\{\left\{\eta_{j}, m_{j}\right\}_{1 \leq j \leq n} \mid n \in \mathbb{N}, \eta_{j}, m_{j}>0, \eta_{i} \neq \eta_{j} \text { if } i \neq j\right\} .
$$

A classical reflectionless potential $u_{\mathbf{s}}$ with scattering data $\mathbf{s}=\left\{\eta_{j}, m_{j}\right\}_{1 \leq j \leq n} \in \mathcal{S}$ is by definition the function

$$
u_{\mathbf{s}}(x)=-2 \frac{d^{2}}{d x^{2}} \log \operatorname{det}\left(I+G_{\mathbf{s}}(x)\right)
$$

where

$$
G_{\mathbf{s}}(x)=\left(\frac{\sqrt{m_{i} m_{j}} e^{-\left(\eta_{i}+\eta_{j}\right) x}}{\eta_{i}+\eta_{j}}\right)_{1 \leq i, j \leq n} .
$$

Applying the inverse scattering theory to the Schrödinger operator $-(d / d x)^{2}+u_{\mathbf{s}}$, one can spell out the scattering data $\mathbf{s}$ from $u_{\mathbf{s}}$. Thus we can identify the space

$$
\Xi_{0}=\left\{u_{\mathbf{s}} \mid \mathbf{s} \in S\right\}
$$

of all classical reflectionless potentials with $\mathcal{S}$. We say that $u$ is a generalized reflectionless potential if there exist $\mu>0$ and $\left\{u_{n}\right\}_{n=1}^{\infty} \subset \Xi_{0}$ such that $u_{n}$ converges to $u$ uniformly on compacts and

$$
\operatorname{Spec}\left(-\frac{d^{2}}{d x^{2}}+u_{n}\right) \subset[-\mu, \infty), \quad n=1,2, \ldots,
$$

where $\operatorname{Spec}\left(-(d / d x)^{2}+u_{n}\right)$ stands for the spectrum of $-(d / d x)^{2}+u_{n}$. Let $\Xi$ be the space of all generalized reflectionless potentials. The space $\Xi$ was used by D. Lundina $[\mathbf{L}]$ and V. Marchenko $[\mathbf{M a 2}]$ to study the Cauchy problem of the KdV equation, and by S. Kotani $[\mathbf{K o 1}, \mathbf{K o 2}]$ to construct the KdV-flow.

Let $\mathcal{W}$ be the space of all continuous functions $w:[0, \infty) \rightarrow \mathbb{R}$ with $w(0)=0$. We denote by $\{X(x)\}_{x \geq 0}$ the coordinate mapping on $\mathcal{W} ; X(x): \mathcal{W} \ni w \mapsto$ $X(x, w)=w(x) \in \mathbb{R}$. Let $\Sigma$ be the space of all finite measures on $\mathbb{R}$ with compact support. For $\sigma \in \Sigma, P^{\sigma}$ denotes the probability measure on $\mathcal{W}$ under which $\{X(x)\}_{x \geq 0}$ is a centered Gaussian process with covariance function

$$
\int_{\mathcal{W}} X(x) X(y) d P^{\sigma}=\int_{\mathbb{R}} \frac{e^{\zeta(x+y)}-e^{\zeta|x-y|}}{2 \zeta} \sigma(d \zeta) .
$$

Set

$$
\mathcal{G}=\left\{P^{\sigma} \mid \sigma \in \Sigma\right\}
$$


Since

$$
\frac{d}{d x} \int_{\mathcal{W}} X(x)^{2} d P^{\sigma}=\int_{\mathbb{R}} e^{2 \zeta x} \sigma(d \zeta)
$$

we may and will identify $\Sigma$ with $\mathcal{G}$.

For $P^{\sigma} \in \mathcal{G}$, we define $\Phi_{\sigma}:[0, \infty) \rightarrow(0, \infty)$ by

$$
\Phi_{\sigma}(x)=\int_{\mathcal{W}} \exp \left(-\frac{1}{2} \int_{0}^{x} X(y)^{2} d y\right) d P^{\sigma}, \quad x \geq 0 .
$$

Then $\Phi_{\sigma} \in C^{\infty}([0, \infty))$ (see $\left.[\mathbf{T 2}]\right)$, and hence we can define $\psi: \mathcal{G} \rightarrow C([0, \infty))$ by

$$
\psi\left(P^{\sigma}\right)(x)=4 \frac{d^{2}}{d x^{2}} \log \Phi_{\sigma}(x), \quad x \geq 0
$$

Put

$$
\Sigma_{0}=\left\{\sigma=\sum_{j=1}^{n} c_{j}^{2} \delta_{p_{j}} \mid n \in \mathbb{N}, c_{j}>0, p_{j} \in \mathbb{R}, j=1, \ldots, n, p_{j} \neq p_{i}(i \neq j)\right\} .
$$

For $\sigma=\sum_{j=1}^{n} c_{j}^{2} \delta_{p_{j}} \in \Sigma_{0}$, we can construct $P^{\sigma}$ by taking advantage of stochastic integrals. Namely, take an $n$-dimensional Brownian motion $\{b(x)\}_{x \geq 0}$ on the probability space $(\Omega, \mathcal{F}, P)$. Representing elements of $\mathbb{R}^{n}$ as column vectors, we define the $\mathbb{R}^{n}$-valued Ornstein-Uhlenbeck process $\left\{\xi_{\sigma}(x)\right\}_{x \geq 0}$ and the $\mathbb{R}$-valued centered Gaussian process $\left\{X_{\sigma}(x)\right\}_{x \geq 0}$ by

$$
\xi_{\sigma}(x)=e^{x D_{\sigma}} \int_{0}^{x} e^{-y D_{\sigma}} d b(y) \quad \text { and } \quad X_{\sigma}(x)=\left\langle\mathbf{c}, \xi_{\sigma}(x)\right\rangle,
$$

respectively, where $D_{\sigma}=\operatorname{diag}\left[p_{j}\right]$ (the diagonal matrix with $p_{1}, \ldots, p_{n}$ being diagonal entries), $e^{A}=\sum_{k=0}^{\infty} A^{k} / k$ ! for $n \times n$-matrix $A,\langle\cdot, \cdot\rangle$ is the standard inner product in $\mathbb{R}^{n}$, and $\mathbf{c}={ }^{t}\left(c_{1}, \ldots, c_{n}\right)$, the transposed vector of $\left(c_{1}, \ldots, c_{n}\right)$. It is easily seen that $P^{\sigma}$ coincides with the induced measure $P \circ X_{\sigma}^{-1}$ on $\mathcal{W}$ through $X_{\sigma}: \Omega \rightarrow \mathcal{W}$.

For $\sigma=\sum_{j=1}^{n} c_{j}^{2} \delta_{p_{j}} \in \Sigma_{0}$, without loss of generality, we may and will assume that there exist $m<n$ and $1 \leq j(1)<\cdots<j(m) \leq n$ such that

$$
\left|p_{j}\right| \leq\left|p_{j+1}\right|, \quad p_{j(\ell)}>0, \quad p_{j(\ell)+1}=-p_{j(\ell)}, \quad \#\left\{\left|p_{1}\right|, \ldots,\left|p_{n}\right|\right\}=n-m .
$$

Let $0<r_{1}<\cdots<r_{n-m}$ be the roots of the algebraic equation

$$
\sum_{j=1}^{n} \frac{c_{j}^{2}}{p_{j}^{2}-r}=-1
$$

We define the mapping $\bar{\psi}: \Sigma_{0} \ni \sigma \mapsto \bar{\psi}(\sigma)=\left\{\eta_{j}, m_{j}\right\} \in \mathcal{S}$ by

$$
\left\{\eta_{1}<\cdots<\eta_{n}\right\}=\left\{p_{j(1)}, \ldots, p_{j(m)}, \sqrt{r_{1}}, \ldots, \sqrt{r_{n-m}}\right\}
$$

and

$$
m_{i}= \begin{cases}2 \eta_{i} \frac{c_{j(\ell)+1}^{2}}{c_{j(\ell)}^{2}} \prod_{k \neq i} \frac{\eta_{k}+\eta_{i}}{\eta_{k}-\eta_{i}} \prod_{k \neq j(\ell), j(\ell)+1} \frac{p_{k}+\eta_{i}}{p_{k}-\eta_{i}}, & \text { if } i=j(\ell), \\ -2 \eta_{i} \prod_{k \neq i} \frac{\eta_{k}+\eta_{i}}{\eta_{k}-\eta_{i}} \prod_{k=1}^{n} \frac{p_{k}+\eta_{i}}{p_{k}-\eta_{i}}, & \text { otherwise. }\end{cases}
$$


Theorem 2.1 ([IkT, T2]). Let $P^{\sigma} \in \mathcal{G}_{0}$ and $\bar{\psi}(\sigma)=\left\{\eta_{j}, m_{j}\right\}_{1 \leq j \leq n}$. (i) It holds that

$$
\begin{aligned}
4 \log \Phi_{\sigma}(x)=-2 \log \operatorname{det}\left(I+G_{\bar{\psi}(\sigma)}(x)\right) & \\
+ & 2 \log \operatorname{det}\left(I+G_{\bar{\psi}(\sigma)}(0)\right)-2 x \sum_{i=1}^{n}\left(p_{i}+\eta_{i}\right), \quad x \geq 0 .
\end{aligned}
$$

(ii) $\psi\left(\mathcal{G}_{0}\right) \subset \Xi_{0}$ and $\psi\left(P^{\sigma}\right)=u_{\bar{\psi}(\sigma)}$.

(iii) $\psi: \mathcal{G}_{0} \rightarrow \Xi_{0}$ is bijective.

The second assertion should be understood as follows. Due to the first assertion, one has that $\psi\left(P^{\sigma}\right)=u_{\bar{\psi}(\sigma)}$ on $[0, \infty)$. Being real analytic on $\mathbb{R}, u_{\bar{\psi}(\sigma)}$ is determined completely by $\psi\left(P^{\sigma}\right)$. In this sense, one thinks of $\psi\left(P^{\sigma}\right)$ as an element in $\Xi_{0}$.

In the proof of $(2.3)$ given in [IkT], the change of variable formula on $\mathcal{W}$, so called the Cameron-Martin formula, played a key role. In the next section, we shall give a new proof of the identity (2.3) by using the filtering theory.

To see the bijectivity described in the assertion (iii), it suffices to construct the inverse mapping of $\bar{\psi}$. For this purpose, let $u=u_{\mathbf{s}} \in \Xi_{0}(\mathbf{s} \in \mathcal{S})$ and $e^{+}(x ; \zeta)$ be the right Jost solution of the Schrödinger operator $\mathcal{L}=-(d / d x)^{2}+u_{\mathbf{s}}$;

$$
\mathcal{L} e^{+}(* ; \zeta)=\zeta^{2} e^{+}(* ; \zeta), \quad e^{+}(x ; \zeta) \sim e^{i \zeta x}(x \rightarrow \infty) .
$$

Then, it is known $([\mathbf{L}, \mathbf{M a 2}])$ that there are $\lambda_{j} \in C^{\infty}(\mathbb{R} ; \mathbb{R}), 1 \leq j \leq n$, such that

$$
e^{+}(x ; \zeta)=e^{\sqrt{-1} \zeta x} \prod_{j=1}^{n} \frac{\zeta-\sqrt{-1} \lambda_{j}(x)}{\zeta+\sqrt{-1} \eta_{j}} \quad \text { and } \quad \lambda_{j}^{\prime}<0,1 \leq j \leq n .
$$

If we set $\kappa(\mathbf{s})=\sum_{j=1}^{n}\left(-\lambda_{j}^{\prime}(0)\right) \delta_{\lambda_{j}(0)}$, then $\kappa=\bar{\psi}^{-1}$.

On account of the above observation on the Jost solution, one can give stochastic expressions on $(-\infty, 0]$;

Theorem $2.2([\mathbf{T} 2])$. Let $P^{\sigma} \in \mathcal{G}_{0}$.

(i) If we define $\mu \in \Sigma_{0}$ by $\mu(A)=\sigma(-A), A \in \mathcal{B}(\mathbb{R})$ (三the Borel field on $\mathbb{R}$ ), then

$$
u_{\bar{\psi}(\sigma)}(x)=\psi\left(P^{\mu}\right)(-x) \quad \text { for } x \in(-\infty, 0] .
$$

(ii) For $y \leq 0$, let $b(y)=b(-y)$. Define

$$
\xi_{\sigma}(y)=-e^{y D_{\sigma}} \int_{y}^{0} e^{-z D_{\sigma}} d b(z), \quad X_{\sigma}(y)=\left\langle\mathbf{c}, \xi_{\sigma}(y)\right\rangle .
$$

Then $u=\psi\left(P^{\sigma}\right)$ is represented as

$$
u(x)=4 \frac{d^{2}}{d x^{2}} \log \left(\int_{\Omega} \exp \left(-\frac{1}{2} \int_{\min \{0, x\}}^{\max \{0, x\}} X_{\sigma}(y)^{2} d y\right) d P\right) \quad \text { for every } x \in \mathbb{R} .
$$

The above bijectivity extends to that between $\mathcal{G}$ and $\Xi$;

Theorem 2.3 ([T2]). (i) Let $\sigma_{n} \in \Sigma_{0}, n=1,2, \ldots$, and $\sigma \in \Sigma$. Assume that $\sigma_{n}$ converges to $\sigma$ vaguely as $n \rightarrow \infty$ and $\bigcup_{n \in \mathbb{N}} \operatorname{supp} \sigma_{n} \subset[-\beta, \beta]$ for some $\beta>0$. Then $\psi\left(P^{\sigma_{n}}\right)$ tends to $\psi\left(P^{\sigma}\right)$ uniformly on compacts in $[0, \infty)$ as $n \rightarrow \infty$. Moreover, for each $\varepsilon>0$, there exists $n_{0} \in \mathbb{N}$ such that

$$
\bigcup_{n \geq n_{0}} \operatorname{Spec}\left(-\frac{d^{2}}{d x^{2}}+\psi\left(P^{\sigma_{n}}\right)\right) \subset\left[-\beta^{2}-\sigma(\mathbb{R})-\varepsilon, \infty\right) .
$$


(ii) Let $\sigma \in \Sigma$, and define $\mu \in \Sigma$ by $\mu(A)=\sigma(-A), A \in \mathcal{B}(\mathbb{R})$. Set

$$
u(x)= \begin{cases}\psi\left(P^{\sigma}\right)(x), & x \geq 0, \\ \psi\left(P^{\mu}\right)(-x), & x \leq 0 .\end{cases}
$$

Then $u \in \Xi$. Conversely every $u \in \Xi$ is of the above form.

For $\sigma \in \Sigma, \sigma_{n}$ 's constructed as follows satisfy the conditions described in (i);

$$
\sigma_{n}=\sum_{j=-n}^{n}\left\{\sigma([j \beta / n,(j+1) \beta / n))+\frac{1}{n}\right\} \delta_{j \beta / n},
$$

where $\beta>0$ has been chosen so that $\operatorname{supp} \sigma \subset[-\beta, \beta]$.

\section{The KdV equation}

As is well known ([MiJD]), soliton solutions of the KdV equation and the $\tau$-function of the $\mathrm{KdV}$ hierarchy are of the same form as classical reflectionless potential. Namely, the $\tau$-function of the KdV hierarchy is given by

$$
\tau(x, \mathbf{t})=\operatorname{det}(I+A(x, \mathbf{t}))
$$

where $x \in \mathbb{R}, \mathbf{t}=\left(t_{1}, t_{2}, \ldots\right) \in \mathbb{R}^{\mathbb{N}}$ satisfies that $\#\left\{t_{j} \neq 0\right\}<\infty,\left\{\eta_{j}, m_{j}\right\} \in \mathcal{S}$, $\zeta_{i}(x, \mathbf{t})=x \eta_{i}+\sum_{\alpha=1}^{\infty} t_{\alpha} \eta_{i}^{2 \alpha+1}$, and

$$
A(x, \mathbf{t})=\left(\frac{\sqrt{m_{i} m_{j}}}{\eta_{i}+\eta_{j}} e^{-\left\{\zeta_{i}(x, \mathbf{t})+\zeta_{j}(x, \mathbf{t})\right\}}\right)_{1 \leq i, j \leq n} .
$$

If $\mathbf{t}=(t, 0, \ldots)$, then $v(x, t)=2(\partial / \partial x)^{2} \log \tau(x, \mathbf{t})$ is a soliton solution of the $\mathrm{KdV}$ equation (1.1) ([MiJD] $)$.

As was seen in [IKT] , we also have a stochastic expression of the $\tau$-function. To recall this, let $\sigma \in \Sigma_{0}$ and $\bar{\psi}(\sigma)=\left\{\eta_{j}, m_{j}\right\} \in \mathcal{S}$. If we denote by $R$ the diagonal matrix $\operatorname{diag}\left[\eta_{j}\right]$ with $\eta_{j}$ 's being the diagonal entries, then there exists $U \in O(n)$ such that $D_{\sigma}^{2}+\mathbf{c} \otimes \mathbf{c}=U R^{2} U^{-1}$, where $\mathbf{c} \otimes \mathbf{c}=\left(c_{i} c_{j}\right)_{1 \leq i, j \leq n} . \operatorname{Set} \zeta(x, \mathbf{t})=\operatorname{diag}\left[\zeta_{j}(x, \mathbf{t})\right]$, and define the $n \times n$-matrix $\phi(x, \mathbf{t})$ by

$$
\phi(x, \mathbf{t})=U\left\{\cosh (\zeta(x, \mathbf{t}))-\sinh (\zeta(x, \mathbf{t})) R^{-1} U^{-1} D_{\sigma} U\right\} U^{-1} .
$$

Then, for every $(x, \mathbf{t}), \operatorname{det} \phi(x, \mathbf{t}) \neq 0$ and one can define the $n \times n$-matrix $\beta_{\mathbf{t}}(x)$ by

Set

$$
\beta_{\mathbf{t}}(x)=-\frac{\partial \phi}{\partial x}(x, \mathbf{t}) \phi^{-1}(x, \mathbf{t}) .
$$

$$
I_{\sigma}(x, \mathbf{t})=\int_{\Omega} \exp \left(-\frac{1}{2} \int_{0}^{x} X_{\sigma}(y)^{2} d y+\frac{1}{2}\left\langle\left(\beta_{\mathbf{t}}(0)-D_{\sigma}\right) \xi_{\sigma}(x), \xi_{\sigma}(x)\right\rangle\right) d P .
$$

We then have that

THEOREM 3.1 ([IkT]). (i) It holds that

$$
\log I_{\sigma}(x, \mathbf{t})=-\frac{1}{2} \log \tau(x, \mathbf{t})+\frac{1}{2} \log \tau(0, \mathbf{t})-\frac{x}{2} \sum_{i=1}^{n}\left(p_{i}+\eta_{i}\right) .
$$

(ii) If $\mathbf{t}=(t, 0, \ldots)$, then the function

$$
v_{\sigma}(x, \mathbf{t})=-4\left(\frac{\partial}{\partial x}\right)^{2} \log I_{\sigma}(x, \mathbf{t})
$$


is an $n$-soliton solution of the KdV equation (1.1).

We next consider the stochastic $\mathrm{KdV}$ equation dealt with in $[\mathbf{W}]$;

$$
d_{t} v-\left\{\frac{3}{2} v \frac{\partial v}{\partial x}+\frac{1}{4} \frac{\partial^{3} v}{\partial x^{3}}\right\} d t=a d W(t)
$$

where $a \in \mathbb{R}$ and $\{W(t)\}_{t \geq 0}$ is a 1-dimensional Brownian motion which is independent of $\{b(x)\}_{x \geq 0}$. For $t \geq 0$, let $\mathbf{t}=(t, 0, \ldots)$ and write $A(x, t)$ and $\beta_{t}(x)$ for $A(x, \mathbf{t})$ and $\beta_{\mathbf{t}}(x)$. Define

$$
\begin{aligned}
I_{\sigma}(x, t ; W)=\int_{\Omega} \exp ( & -\frac{1}{2} \int_{0}^{x} X_{\sigma}(y)^{2} d y \\
& \left.+\frac{1}{2}\left\langle\left\{\beta_{t}\left(-\frac{3 a}{2} \int_{0}^{t} W(u) d u\right)-D_{\sigma}\right\} \xi_{\sigma}(x), \xi_{\sigma}(x)\right\rangle\right) d P .
\end{aligned}
$$

Then we have that

THEOREM 3.2. The function given by

$$
v(x, t ; W)=a W(t)-4\left(\frac{\partial}{\partial x}\right)^{2} \log I_{\sigma}(x, t ; W)
$$

is a solution of the stochastic KdV equation (3.1).

Proof. In repetition of the argument in $[\mathbf{I k T}]$, we have that

$$
\begin{aligned}
& \log \left(\int_{\Omega} \exp \left(-\frac{1}{2} \int_{0}^{x} X_{\sigma}(y)^{2} d y+\frac{1}{2}\left\langle\left\{\beta_{t}\left(x_{0}\right)-D_{\sigma}\right\} \xi_{\sigma}(x), \xi_{\sigma}(x)\right\rangle\right) d P\right) \\
& =-\frac{1}{2} \log \operatorname{det}\left(I+A\left(x+x_{0}, t\right)\right)+\frac{1}{2} \log \operatorname{det}\left(I+A\left(x_{0}, t\right)\right)-\frac{x}{2} \sum_{i=1}^{n}\left(p_{i}+\eta_{i}\right)
\end{aligned}
$$

for any $x_{0} \in \mathbb{R}$. While the stochastic KdV equation dealt with by Wadati $[\mathbf{W}]$ was of the form

$$
d_{t} v-\left\{6 v \frac{\partial v}{\partial x}-\frac{\partial^{3} v}{\partial x^{3}}\right\} d t=d W(t)
$$

after the standard change of variables, his result is rewritten in our setting as follows; the function

$$
v(x, t ; W)=a W(t)+2\left(\frac{\partial}{\partial x}\right)^{2} \log \left(\operatorname{det}\left[I+A\left(x-\frac{3 a}{2} \int_{0}^{t} W(u) d u, t\right)\right]\right)
$$

solves the stochastic KdV equation (3.1). Combining this with (3.2), we arrive at the desired assertion.

\section{The filtering theory}

The results in the previous sections, which connects the Gaussian measures and reflectionless potentials and the KdV equation, start from the identity (2.3). In $[\mathbf{I k T}]$, the identity was shown by using the Cameron-Martin formula on the Wiener space. In this section, we first give an alternative proof of (2.3) with the help of the linear filtering theory. Secondly we investigate $\Phi_{\sigma}$ from the Gaussian filtering theoretical point of view to revisit the Marchenko formula. 
4.1. Proof of (2.3) via the linear filtering theory. Let $\sigma=\sum_{j=1}^{n} c_{j}^{2} \delta_{p_{j}} \in$ $\Sigma_{0}$. Throughout this subsection, we assume that

$$
\left|p_{1}\right|<\cdots<\left|p_{n}\right| .
$$

The general case follows from this one in the standard limiting procedure as was seen in [IkT]. Let $\{b(x)\}_{x>0}$ be an $n$-dimensional Brownian motion on a filtered probability space $\left(\Omega, \mathcal{F}, P,\left\{\mathcal{F}_{x}\right\}_{x \geq 0}\right)$, and define $\left\{\xi_{\sigma}(x)={ }^{t}\left(\xi_{\sigma}^{1}(x), \ldots, \xi_{\sigma}^{n}(x)\right)\right\}_{x \geq 0}$ and $\left\{X_{\sigma}(x)\right\}_{x \geq 0}$ as in Section 2 .

Take a 1-dimensional Brownian motion $\{N(x)\}_{x \geq 0}$ with $N(0)=0$, which is independent of $\{b(x)\}_{x \geq 0}$, and set

$$
Z_{\sigma}(x)=\int_{0}^{x} X_{\sigma}(y) d y+N(x)
$$

$\mathcal{Z}_{x}=\sigma\left[Z_{\sigma}(y) ; y \leq x\right], \widehat{X}_{\sigma}(x)=\mathbb{E}\left[X_{\sigma}(x) \mid \mathcal{Z}_{x}\right]$, the conditional expectation of $X_{\sigma}(x)$ given $\mathcal{Z}_{x}$, and

$$
S_{\sigma}(x)=\int_{\Omega}\left(X_{\sigma}(x)-\widehat{X}_{\sigma}(x)\right)^{2} d P .
$$

It was shown by Kleptsyna-Le Breton $[$ KlB $]$ that

$$
\int_{\Omega} \exp \left(-\frac{1}{2} \int_{0}^{x} X_{\sigma}(y)^{2} d y\right) d P=\exp \left(-\frac{1}{2} \int_{0}^{x} S_{\sigma}(y) d y\right) .
$$

Combined with the definition of $X_{\sigma}(x)$, this implies that

$$
\log \left(\int_{\Omega} \exp \left(-\frac{1}{2} \int_{0}^{x} X_{\sigma}(y)^{2} d y\right) d P\right)=-\frac{1}{2} \int_{0}^{x}\left(\sum_{i, j=1}^{n} c_{i} c_{j} P_{i j}(y)\right) d y
$$

where

$$
P_{i j}(y)=\int_{\Omega}\left(\xi_{\sigma}^{i}(y)-\widehat{\xi_{\sigma}^{i}}(y)\right)\left(\xi_{\sigma}^{j}(y)-\widehat{\xi_{\sigma}^{j}}(y)\right) d P .
$$

Since the $n \times n$-matrix valued function $P=\left(P_{i j}\right)$ is the error matrix of the linear filtering problem

$$
\begin{cases}d \xi_{\sigma}(x)=D_{\sigma} \xi_{\sigma}(x) d x+d b(x) & \text { (system) } \\ d Z_{\sigma}(x)=\left\langle\mathbf{c}, \xi_{\sigma}(x)\right\rangle d x+d N(x) & \text { (observation) }\end{cases}
$$

it obeys the ordinary differential equation

$$
\frac{d P}{d x}(x)=D_{\sigma} P(x)+P(x) D_{\sigma}-P(x)(\mathbf{c} \otimes \mathbf{c}) P(x)+I, \quad P(0)=0,
$$

where $I$ denotes the $n \times n$ unit matrix. See $[\mathbf{B J}]$. Thus, what is needed to show (2.3) is a precise expression of $\sum_{i, j=1}^{n} c_{i} c_{j} P_{i j}(x)$.

Put

$$
H=\left(\begin{array}{cc}
-D_{\sigma} & \mathbf{c} \otimes \mathbf{c} \\
I & D_{\sigma}
\end{array}\right) \quad \text { and } \quad\left(\begin{array}{c}
U_{0}(x) \\
V_{0}(x)
\end{array}\right)=\exp (x H)\left(\begin{array}{l}
I \\
0
\end{array}\right),
$$

where 0 denotes the $n \times n$ zero matrix. Since

$$
\left\langle U_{0}(x) v, V_{0}(x) v\right\rangle=\int_{0}^{x}\left\{\left|U_{0}(y) v\right|^{2}+\left\langle\mathbf{c}, V_{0}(y) v\right\rangle^{2}\right\} d y, \quad v \in \mathbb{R}^{n},
$$

we see that $\operatorname{det} U_{0}(x) \neq 0$ for any $x \geq 0$. It is then easily checked that

$$
P(x)=V_{0}(x) U_{0}(x)^{-1} \text {. }
$$


Let $0<r_{1}<\cdots<r_{n}$ be the roots of $(2.1)$, and set $\eta_{j}=\sqrt{r_{j}}$. Define $n \times n$-matrices $A, B, C$ and $(2 n) \times(2 n)$-matrix $S$ by

$$
\begin{aligned}
& A=\left(\left(D_{\sigma}+\eta_{j}\right)^{-1} \mathbf{c}\right)_{1 \leq j \leq n}, \quad B=\left(\left(D_{\sigma}-\eta_{j}\right)^{-1} \mathbf{c}\right)_{1 \leq j \leq n}, \\
& C=\left(-\left(D_{\sigma}^{2}-r_{j}\right)^{-1} \mathbf{c}\right)_{1 \leq j \leq n}, \quad S=\left(\begin{array}{cc}
A & B \\
C & C
\end{array}\right) .
\end{aligned}
$$

Then $\operatorname{det} S \neq 0$ and it holds that

$$
H=S\left(\begin{array}{cc}
R & 0 \\
0 & -R
\end{array}\right) S^{-1} .
$$

Since

$$
S=\left(\begin{array}{cc}
A-B & B \\
0 & C
\end{array}\right)\left(\begin{array}{ll}
I & 0 \\
I & I
\end{array}\right),
$$

$\operatorname{det}(A-B) \neq 0$ and $\operatorname{det} C \neq 0$. We then have that

$$
\exp (x H)=\left(\begin{array}{ll}
A e^{x R} & B e^{-x R} \\
C e^{x R} & C e^{-x R}
\end{array}\right)\left(\begin{array}{cc}
(A-B)^{-1} & -(A-B)^{-1} B C^{-1} \\
-(A-B)^{-1} & (A-B)^{-1} B C^{-1}+C^{-1}
\end{array}\right) .
$$

Due to (4.3), this implies that

$$
P(x)=C\left(e^{x R}-e^{-x R}\right)\left\{A e^{x R}-B e^{-x R}\right\}^{-1} .
$$

Define $n \times n$-matrices $X, Y, Z$ by

$$
X=\left(\frac{1}{p_{i}+\eta_{j}}\right)_{1 \leq i, j \leq n}, \quad Y=\left(\frac{1}{p_{i}-\eta_{j}}\right)_{1 \leq i, j \leq n}, \quad Z=\left(\frac{1}{p_{i}^{2}-r_{j}}\right)_{1 \leq i, j \leq n} .
$$

Since $A=\operatorname{diag}\left[c_{j}\right] X, B=\operatorname{diag}\left[c_{j}\right] Y, C=-\operatorname{diag}\left[c_{j}\right] Z$, we obtain that

$$
P(x)=-\operatorname{diag}\left[c_{j}\right] Z\left(e^{x R}-e^{-x R}\right)\left\{X e^{x R}-Y e^{-x R}\right\}^{-1} \operatorname{diag}\left[1 / c_{j}\right] .
$$

In conjunction with the definition of $r_{k}$ 's, this yields that

$$
\sum_{i, j=1}^{n} c_{i} c_{j} P_{i j}(x)=\sum_{i, j=1}^{n} Q_{i j}(x)
$$

where

$$
Q(x)=\left(I-e^{-2 x R}\right)\left\{X-Y e^{-2 x R}\right\}^{-1} .
$$

In the sequel, we compute $\sum_{i, j=1}^{n} Q_{i j}(x)$ algebraically, and do not use the dependence of $\eta_{j}$ 's on $p_{i}$ 's. Hence, in what follows we only assume that $p_{i} \neq p_{j}$ and $\eta_{i} \neq \eta_{j}$ if $i \neq j, \eta_{j}>0,1 \leq j \leq n$, and $\left\{p_{1}, \ldots, p_{n}\right\} \cap\left\{\eta_{1}, \ldots \eta_{n}\right\}=\emptyset$. Define $m_{i}>0$ by $(2.2)$;

$$
m_{i}=-2 \eta_{i} \prod_{k \neq i} \frac{\eta_{k}+\eta_{i}}{\eta_{k}-\eta_{i}} \prod_{k=1}^{n} \frac{p_{k}+\eta_{i}}{p_{k}-\eta_{i}}
$$

If we set $T(\zeta)=X-Y \operatorname{diag}\left[\zeta_{j}^{2}\right], \zeta={ }^{t}\left(\zeta_{1}, \ldots, \zeta_{n}\right) \in \mathbb{R}^{n}$, then

$$
T\left({ }^{t}\left(e^{-x \eta_{1}}, \ldots, e^{-x \eta_{n}}\right)\right)=X-Y e^{-2 x R}
$$

and

$$
\operatorname{det} T(\zeta)=\operatorname{det} X+\sum_{p=1}^{n} \sum_{1 \leq j_{1}<\cdots<j_{p} \leq n}\left(\operatorname{det} X_{j_{1} \ldots j_{p}}\right) \zeta_{j_{1}}^{2} \ldots \zeta_{j_{p}}^{2},
$$


where $X_{j_{1} \ldots j_{p}}$ is the matrix obtained by replacing all $j_{k}$-th column, $1 \leq k \leq p$, of $X$ by $^{t}\left(-1 /\left(p_{i}-\eta_{j_{k}}\right)\right)_{1 \leq i \leq n}, 1 \leq k \leq p$, respectively. Due to Cauchy's identity

$$
\operatorname{det}\left[\left(\frac{1}{\alpha_{i}+\beta_{j}}\right)_{1 \leq i, j \leq n}\right]=\frac{\prod_{1 \leq i<j \leq n}\left(\alpha_{i}-\alpha_{j}\right)\left(\beta_{i}-\beta_{j}\right)}{\prod_{i, j=1}^{n}\left(\alpha_{i}+\beta_{j}\right)},
$$

we obtain that

$$
\operatorname{det} X_{j_{1} \ldots j_{p}}=\prod_{k=1}^{p} \frac{m_{j_{k}}}{2 \eta_{j_{k}}} \prod_{1 \leq k<\ell<p}\left(\frac{\eta_{j_{k}}-\eta_{j_{\ell}}}{\eta_{j_{k}}+\eta_{j_{\ell}}}\right)^{2} \times \operatorname{det} X .
$$

Since

$$
\begin{aligned}
& \operatorname{det}\left(I+G_{\mathbf{s}}(x)\right) \\
& =1+\sum_{p=1}^{n} \sum_{1 \leq i_{1}<\cdots<i_{p} \leq n} \prod_{j=1}^{p} \frac{m_{i_{j}}}{2 \eta_{i_{j}}} \prod_{1 \leq j<k \leq p}\left(\frac{\eta_{i_{j}}-\eta_{i_{k}}}{\eta_{i_{j}}+\eta_{i_{k}}}\right)^{2} \exp \left(-2 x \sum_{j=1}^{p} \eta_{i_{j}}\right),
\end{aligned}
$$

we have that

$$
\operatorname{det}\left(X-Y e^{-2 x R}\right)=\operatorname{det} X \cdot \operatorname{det}\left(I+G_{\mathbf{s}}(x)\right) .
$$

Denote by $\widetilde{T}(\zeta)$ the cofactor matrix of $T(\zeta)$, and set $\widehat{Q}(\zeta)=\left(I-\operatorname{diag}\left[\zeta_{j}^{2}\right]\right) \widetilde{T}(\zeta)$. It then holds that

$$
Q(x)=\left.\frac{1}{\operatorname{det} T(\zeta)} \widehat{Q}(\zeta)\right|_{\zeta==^{t}\left(e^{-x \eta_{1}}, \ldots, e^{-x \eta_{n}}\right)} .
$$

If we use $T_{<i>}(\zeta)$ to indicate the matrix obtained by replacing the $i$-th line of $T(\zeta)$ by $\left(1-\zeta_{1}^{2}, \ldots, 1-\zeta_{n}^{2}\right)$, then it holds that

$$
\sum_{k, i=1}^{n} \widehat{Q}_{k i}(\zeta)=\sum_{k, i=1}^{n}\left(1-\zeta_{k}^{2}\right) \widetilde{T}_{k i}(\zeta)=\sum_{i=1}^{n} \operatorname{det} T_{<i>}(\zeta) .
$$

Since $\lim _{p_{i} \rightarrow \infty} p_{i} T_{i k}(\zeta)=1-\zeta_{k}^{2}, 1 \leq k \leq n$, we observe that

$$
\operatorname{det} T_{<i>}(\zeta)=\lim _{p_{i} \rightarrow \infty} p_{i} \operatorname{det} T(\zeta)=\sum_{p=0}^{n} \sum_{1 \leq j_{1}<\cdots<j_{p} \leq n}\left(\operatorname{det} X_{j_{1} \ldots j_{p} ; i}\right) \zeta_{j_{1}}^{2} \ldots \zeta_{j_{p}}^{2},
$$

where $X_{j_{1} \ldots j_{p} ; i}=\left(\left(X_{j_{1} \ldots j_{p} ; i}\right)_{k \ell}\right)_{1 \leq k, \ell \leq n}$ is given by

$$
\left(X_{j_{1} \ldots j_{p} ; i}\right)_{k \ell}= \begin{cases}1 /\left(p_{k}+\eta_{\ell}\right), & k \neq i, \ell \notin\left\{j_{1}, \ldots, j_{p}\right\}, \\ -1 /\left(p_{k}-\eta_{\ell}\right), & k \neq i, \ell \in\left\{j_{1}, \ldots, j_{p}\right\}, \\ 1, & k=i, \ell \notin\left\{j_{1}, \ldots, j_{p}\right\}, \\ -1, & k=i, \ell \in\left\{j_{1}, \ldots, j_{p}\right\} .\end{cases}
$$

In the above expression, for $p=0, \zeta_{j_{1}}^{2} \ldots \zeta_{j_{p}}^{2}=1$ and the summation $\sum_{1 \leq j_{1}<\cdots<j_{p} \leq n}$ is just one term determined as $\left\{j_{1}, \ldots, j_{p}\right\}=\emptyset$. Thus we obtain that

$$
\sum_{i, j=1}^{n} \widehat{Q}_{i j}(\zeta)=\sum_{p=0}^{n} \sum_{1 \leq j_{1}<\cdots<j_{p} \leq n}\left(\sum_{i=1}^{n} \operatorname{det} X_{j_{1} \ldots j_{p} ; i}\right) \zeta_{j_{1}}^{2} \ldots \zeta_{j_{p}}^{2} .
$$

For

$$
K=\left(\frac{1}{\alpha_{i}+\beta_{j}}\right)_{1 \leq i, j \leq n}
$$


and its cofactor matrix $\widetilde{K}$, it holds that

Hence if we set

$$
\sum_{i, j=1}^{n} \widetilde{K}_{i j}=\operatorname{det} K \sum_{i=1}^{n}\left(\alpha_{i}+\beta_{i}\right)
$$

$$
\left(X_{j_{1} \ldots j_{p}}^{\prime}\right)_{k \ell}= \begin{cases}\frac{1}{p_{k}+\eta_{\ell}}, & \ell \notin\left\{j_{1}, \ldots, j_{p}\right\} \\ \frac{1}{p_{k}-\eta_{\ell}}, & \ell \in\left\{j_{1}, \ldots, j_{p}\right\}\end{cases}
$$

and denote by $\widetilde{X_{j_{1} \ldots j_{p}}^{\prime}}$ the cofactor matrix of $X_{j_{1} \ldots j_{p}}^{\prime}$, then, by (4.5), we have that

$$
\begin{aligned}
& \sum_{i=1}^{n} \operatorname{det} X_{j_{1} \ldots j_{p} ; i}=(-1)^{p} \sum_{k, \ell=1}^{n}\left(\widetilde{X_{j_{1} \ldots j_{p}}^{\prime}}\right)_{k \ell} \\
& =(-1)^{p}\left\{A-2 \sum_{k=1}^{p} \eta_{j_{k}}\right\} \operatorname{det} X_{j_{1} \ldots j_{p}}^{\prime}=\left\{A-2 \sum_{k=1}^{p} \eta_{j_{k}}\right\} \operatorname{det} X_{j_{1} \ldots j_{p}} \\
& \quad=\left\{A-2 \sum_{k=1}^{p} \eta_{j_{k}}\right\} \operatorname{det} X \prod_{k=1}^{p} \frac{m_{j_{k}}}{2 \eta_{j_{k}}} \prod_{1 \leq k<\ell \leq p}\left(\frac{\eta_{j_{k}}-\eta_{j_{\ell}}}{\eta_{j_{k}}+\eta_{j_{\ell}}}\right)^{2},
\end{aligned}
$$

where $A=\sum_{i=1}^{n}\left(p_{i}+\eta_{i}\right)$. Plugging this into (4.9), we obtain that

$$
\begin{aligned}
\sum_{i, j=1}^{n} \widehat{Q}_{i j}(\zeta)=\operatorname{det} X\left\{A+\sum_{p=1}^{n} \sum_{1 \leq j_{1}<\cdots<j_{p} \leq n}\left\{A-2 \sum_{k=1}^{p} \eta_{j_{k}}\right\} \times\right. \\
\left.\times \prod_{k=1}^{p} \frac{m_{j_{k}}}{2 \eta_{j_{k}}} \prod_{1 \leq k<\ell \leq p}\left(\frac{\eta_{j_{k}}-\eta_{j_{\ell}}}{\eta_{j_{k}}+\eta_{j_{\ell}}}\right)^{2} \zeta_{j_{1}}^{2} \ldots \zeta_{j_{p}}^{2}\right\}
\end{aligned}
$$

Combining this with (4.6), (4.7) and (4.8), we arrive at

$$
\sum_{i, j=1}^{n} Q_{i j}(x)=\sum_{i=1}^{n}\left(p_{i}+\eta_{i}\right)+\frac{d}{d x} \log \operatorname{det}\left(I+G_{\mathbf{s}}(x)\right) .
$$

From this, (4.1) and (4.4), the identity (2.3) follows.

4.2. The Gaussian filtering theory. In this subsection, we give a probabilistic interpretation of the Marchenko formula ([Ma1]) in the inverse scattering theory through the Gaussian filtering theory.

We start with recalling the Gaussian filtering theory. Let $u \in \Xi$ and, as an application of Theorem 2.3, take $P^{\sigma} \in \mathcal{G}$ so that $u=\psi\left(P^{\sigma}\right)$ on $[0, \infty)$. Let $\Omega=\mathcal{W} \times \mathcal{W}$, and define the probability measure $P=P^{\sigma} \times P^{\delta_{0}}$ on $\Omega$. Notice that $P^{\delta_{0}}$ is the Wiener measure on $\mathcal{W}$. Denote by $\{(X(x), N(x))\}_{x \geq 0}$ the coordinate mapping of $\Omega ; X(x, w)=w^{1}(x), N(x, w)=w^{2}(x)$ for $w=\left(w^{1}, w^{2}\right) \in \Omega$. Define

$$
Z(x)=\int_{0}^{x} X(y) d y+N(x), \quad x \geq 0 .
$$

Set $\mathcal{Z}_{x}=\sigma[Z(y): y \leq x], \widehat{X}(x)=\mathbb{E}\left[X(x) \mid \mathcal{Z}_{x}\right]$, and

$$
S(x)=\int_{\Omega}(X(x)-\widehat{X}(x))^{2} d P .
$$


Let $K(x, y)$ be the unique Volterra kernel solving the Wiener-Hopf type equation

$$
K(x, y)+\int_{0}^{x} K(x, z) R_{\sigma}(z, y) d z=R_{\sigma}(x, y),
$$

where $R_{\sigma}(x, y)=\int_{\mathcal{W}} X(x) X(y) d P^{\sigma}$, and $L(x, y)$ be the unique Volterra kernel to the resolvent equation

$$
L(x, y)-K(x, y)-\int_{y}^{x} K(x, z) L(z, y) d z=0 .
$$

Then it holds that

$$
L(x, y)= \begin{cases}\int_{\Omega} X(x)(X(y)-\widehat{X}(y)) d P, & x \geq y, \\ 0, & x<y\end{cases}
$$

and

$$
S(x)=L(x, x)=K(x, x) .
$$

It was shown by Kleptsyna-Le Breton $[\mathbf{K l B}]$ that

$$
\int_{\Omega} \exp \left(-\frac{1}{2} \int_{0}^{x} X(y)^{2} d y\right) d P=\exp \left(-\frac{1}{2} \int_{0}^{x} S(y) d y\right) .
$$

Hence we have that

$$
u(x)=\psi\left(P^{\sigma}\right)(x)=2 \frac{d}{d x}(-K(x, x)), \quad x \geq 0 .
$$

This identity is exactly the Marchenko formula in the inverse scattering theory ([Ma1]). Thus we have revisited the Marchenko formula via the stochastic calculus.

\section{References}

[BJ] R. Bucy and P. Joseph, Filtering for stochastic processes with applications to guidance, Intersci. Publ., 1968.

[CM] R.H. Cameron and W.T. Martin, The Wiener measure of Hilbert neighbourhoods in the space of real continuous functions, J. Math. Phys. M.I.T., 23 (1944), 195-209.

[IkT] N. Ikeda, S. Taniguchi, Quadratic Wiener functionals, Kalman-Bucy filters, and the KdV equation, in: H. Kunita, S. Watanabe, Y. Takahashi (Eds.) Stochastic Analysis and Related Topics in Kyoto, In honor of Kiyosi Itô, Adv. Studies Pure Math. 41, Math. Soc. Japan, Tokyo, 2004, pp. 167-187.

[It] K. Itô, Differential equations determining a Markoff process (in Japanese), Journ. PanJapan Math. Coll. No.1077 (1942). English transl. in "Kiyosi Itô Selected papers" ed. by D. Stroock and S.R.S. Varadhan, Springer, 1987, 42-75.

[Ka] M. Kac, On distributions of certain Wiener functionals, Trans. Amer. Math. Soc. 65 (1949), 1-13.

[KlB] M.L. Kleptsyna and A. Le Breton, A Cameron-Martin type formula for general Gaussian processes - A filtering approach, Stoch. Stoch. Rep., 72 (2002), 229-250.

[Ko1] S. Kotani, Probabilistic approach to reflectionless potentials (in Japanese), Symposium on Random matrices and related topics at Tsukuba Univ., 2000.

[Ko2] S. Kotani, Construction of KdV-flow on generalized reflectionless potentials, preprint (2003 November)

[L] D.Sh. Lundina, Compactness of the set of reflection-free potentials, Teor. Funk. Funk. Anal. Ikh Pri. 44 (1985) 57-66, English transl. J. Soviet Math. 48 (1990) 290-297.

[Ma1] V.A. Marchenko, Sturm-Liouville operators and applications, Birkhäuser, Boston, 1986.

[Ma2] V.A. Marchenko, The Cauchy problem for the KdV equation with non-decreasing initial data, in: Zakharov, V.E. (Ed.) What is integrability? Springer-Verlag, New York, 1990, pp. 273-318.

[MiJD] T. Miwa, M. Jimbo and E. Date, Solitons, Cambridge Univ. Press, Cambridge, 2000. 
[T1] S. Taniguchi, On Wiener functionals of order 2 associated with soliton solutions of the KdV equation, Jour. Funct. Anal. 216 (2004) 212-229.

[T2] S. Taniguchi, Brownian sheet and reflectionless potentials, Stoch. Proc. their Appl. 116 (2006), 293-309.

[W] M. Wadati, Stochastic Korteweg-de Vries Equation, J. Phys. Soc. Japan 52 (1983), 26422648 .

Faculty Of Mathematics, Kyushu University, FukUOKa 812-8581, Japan

E-mail address: taniguch@math.kyushu-u.ac.jp 


\section{List of MHF Preprint Series, Kyushu University 21st Century COE Program \\ Development of Dynamic Mathematics with High Functionality}

\section{MHF2005-1 Hideki KOSAKI}

Matrix trace inequalities related to uncertainty principle

MHF2005-2 Masahisa TABATA

Discrepancy between theory and real computation on the stability of some finite element schemes

MHF2005-3 Yuko ARAKI \& Sadanori KONISHI

Functional regression modeling via regularized basis expansions and model selection

MHF2005-4 Yuko ARAKI \& Sadanori KONISHI

Functional discriminant analysis via regularized basis expansions

MHF2005-5 Kenji KAJIWARA, Tetsu MASUDA, Masatoshi NOUMI, Yasuhiro OHTA \& Yasuhiko YAMADA

Point configurations, Cremona transformations and the elliptic difference Painlevé equations

MHF2005-6 Kenji KAJIWARA, Tetsu MASUDA, Masatoshi NOUMI, Yasuhiro OHTA \& Yasuhiko YAMADA

Construction of hypergeometric solutions to the $q$ - Painlevé equations

MHF2005-7 Hiroki MASUDA

Simple estimators for non-linear Markovian trend from sampled data:

I. ergodic cases

MHF2005-8 Hiroki MASUDA \& Nakahiro YOSHIDA

Edgeworth expansion for a class of Ornstein-Uhlenbeck-based models

MHF2005-9 Masayuki UCHIDA

Approximate martingale estimating functions under small perturbations of dynamical systems

MHF2005-10 Ryo MATSUZAKI \& Masayuki UCHIDA

One-step estimators for diffusion processes with small dispersion parameters from discrete observations

MHF2005-11 Junichi MATSUKUBO, Ryo MATSUZAKI \& Masayuki UCHIDA

Estimation for a discretely observed small diffusion process with a linear drift

MHF2005-12 Masayuki UCHIDA \& Nakahiro YOSHIDA

AIC for ergodic diffusion processes from discrete observations 
MHF2005-13 Hiromichi GOTO \& Kenji KAJIWARA

Generating function related to the Okamoto polynomials for the Painlevé IV equation

MHF2005-14 Masato KIMURA \& Shin-ichi NAGATA

Precise asymptotic behaviour of the first eigenvalue of Sturm-Liouville problems with large drift

MHF2005-15 Daisuke TAGAMI \& Masahisa TABATA

Numerical computations of a melting glass convection in the furnace

MHF2005-16 Raimundas VIDŪNAS

Normalized Leonard pairs and Askey-Wilson relations

MHF2005-17 Raimundas VIDŪNAS

Askey-Wilson relations and Leonard pairs

MHF2005-18 Kenji KAJIWARA \& Atsushi MUKAIHIRA

Soliton solutions for the non-autonomous discrete-time Toda lattice equation

MHF2005-19 Yuu HARIYA

Construction of Gibbs measures for 1-dimensional continuum fields

MHF2005-20 Yuu HARIYA

Integration by parts formulae for the Wiener measure restricted to subsets in $\mathbb{R}^{d}$

MHF2005-21 Yuu HARIYA

A time-change approach to Kotani's extension of Yor's formula

MHF2005-22 Tadahisa FUNAKI, Yuu HARIYA \& Mark YOR

Wiener integrals for centered powers of Bessel processes, I

MHF2005-23 Masahisa TABATA \& Satoshi KAIZU

Finite element schemes for two-fluids flow problems

MHF2005-24 Ken-ichi MARUNO \& Yasuhiro OHTA

Determinant form of dark soliton solutions of the discrete nonlinear Schrödinger equation

MHF2005-25 Alexander V. KITAEV \& Raimundas VIDŪNAS

Quadratic transformations of the sixth Painlevé equation

MHF2005-26 Toru FUJII \& Sadanori KONISHI

Nonlinear regression modeling via regularized wavelets and smoothing parameter selection

MHF2005-27 Shuichi INOKUCHI, Kazumasa HONDA, Hyen Yeal LEE, Tatsuro SATO, Yoshihiro MIZOGUCHI \& Yasuo KAWAHARA

On reversible cellular automata with finite cell array 
MHF2005-28 Toru KOMATSU

Cyclic cubic field with explicit Artin symbols

MHF2005-29 Mitsuhiro T. NAKAO, Kouji HASHIMOTO \& Kaori NAGATOU

A computational approach to constructive a priori and a posteriori error estimates for finite element approximations of bi-harmonic problems

MHF2005-30 Kaori NAGATOU, Kouji HASHIMOTO \& Mitsuhiro T. NAKAO Numerical verification of stationary solutions for Navier-Stokes problems

MHF2005-31 Hidefumi KAWASAKI

A duality theorem for a three-phase partition problem

MHF2005-32 Hidefumi KAWASAKI

A duality theorem based on triangles separating three convex sets

MHF2005-33 Takeaki FUCHIKAMI \& Hidefumi KAWASAKI

An explicit formula of the Shapley value for a cooperative game induced from the conjugate point

MHF2005-34 Hideki MURAKAWA

A regularization of a reaction-diffusion system approximation to the two-phase Stefan problem

MHF2006-1 Masahisa TABATA

Numerical simulation of Rayleigh-Taylor problems by an energy-stable finite element scheme

MHF2006-2 Ken-ichi MARUNO \& G R W QUISPEL

Construction of integrals of higher-order mappings

MHF2006-3 Setsuo TANIGUCHI

On the Jacobi field approach to stochastic oscillatory integrals with quadratic phase function

MHF2006-4 Kouji HASHIMOTO, Kaori NAGATOU \& Mitsuhiro T. NAKAO

A computational approach to constructive a priori error estimate for finite element approximations of bi-harmonic problems in nonconvex polygonal domains

MHF2006-5 Hidefumi KAWASAKI

A duality theory based on triangular cylinders separating three convex sets in $R^{n}$

MHF2006-6 Raimundas VIDŪNAS

Uniform convergence of hypergeometric series

MHF2006-7 Yuji KODAMA \& Ken-ichi MARUNO

N-Soliton solutions to the DKP equation and Weyl group actions 
MHF2006-8 Toru KOMATSU

Potentially generic polynomial

MHF2006-9 Toru KOMATSU

Generic sextic polynomial related to the subfield problem of a cubic polynomial

MHF2006-10 Shu TEZUKA \& Anargyros PAPAGEORGIOU

Exact cubature for a class of functions of maximum effective dimension

MHF2006-11 Shu TEZUKA

On high-discrepancy sequences

MHF2006-12 Raimundas VIDŪNAS

Detecting persistent regimes in the North Atlantic Oscillation time series

MHF2006-13 Toru KOMATSU

Tamely Eisenstein field with prime power discriminant

MHF2006-14 Nalini JOSHI, Kenji KAJIWARA \& Marta MAZZOCCO

Generating function associated with the Hankel determinant formula for the solutions of the Painlevé IV equation

MHF2006-15 Raimundas VIDŪNAS

Darboux evaluations of algebraic Gauss hypergeometric functions

MHF2006-16 Masato KIMURA \& Isao WAKANO

New mathematical approach to the energy release rate in crack extension

MHF2006-17 Toru KOMATSU

Arithmetic of the splitting field of Alexander polynomial

MHF2006-18 Hiroki MASUDA

Likelihood estimation of stable Lévy processes from discrete data

MHF2006-19 Hiroshi KAWABI \& Michael RÖCKNER

Essential self-adjointness of Dirichlet operators on a path space with Gibbs measures via an SPDE approach

MHF2006-20 Masahisa TABATA

Energy stable finite element schemes and their applications to two-fluid flow problems

MHF2006-21 Yuzuru INAHAMA \& Hiroshi KAWABI

Asymptotic expansions for the Laplace approximations for Itô functionals of Brownian rough paths

MHF2006-22 Yoshiyuki KAGEI

Resolvent estimates for the linearized compressible Navier-Stokes equation in an infinite layer 
MHF2006-23 Yoshiyuki KAGEI

Asymptotic behavior of the semigroup associated with the linearized compressible Navier-Stokes equation in an infinite layer

MHF2006-24 Akihiro MIKODA, Shuichi INOKUCHI, Yoshihiro MIZOGUCHI \& Mitsuhiko FUJIO

The number of orbits of box-ball systems

MHF2006-25 Toru FUJII \& Sadanori KONISHI

Multi-class Logistic Discrimination via Wavelet-based Functionalization and Model Selection Criteria

MHF2006-26 Taro HAMAMOTO, Kenji KAJIWARA \& Nicholas S. WITTE

Hypergeometric Solutions to the $q$-Painlevé Equation of Type $\left(A_{1}+A_{1}^{\prime}\right)^{(1)}$

MHF2006-27 Hiroshi KAWABI \& Tomohiro MIYOKAWA

The Littlewood-Paley-Stein inequality for diffusion processes on general metric spaces

MHF2006-28 Hiroki MASUDA

Notes on estimating inverse-Gaussian and gamma subordinators under highfrequency sampling

MHF2006-29 Setsuo TANIGUCHI

The heat semigroup and kernel associated with certain non-commutative harmonic oscillators

MHF2006-30 Setsuo TANIGUCHI

Stochastic analysis and the KdV equation 